\title{
Linx
}

Revue des linguistes de l'université Paris X Nanterre

$11 \mid 1999$

Typologie des langues, universaux linguistiques

\section{Typologies phonologiques et tendances universelles. Approche substantialiste}

Nathalie Vallée, Louis-Jean Boë et Muriel Stefanuto

\section{(2) OpenEdition}

\section{Journals}

Édition électronique

URL : http://journals.openedition.org/linx/863

DOI : $10.4000 / \operatorname{lin} x .863$

ISSN : 2118-9692

Éditeur

Presses universitaires de Paris Nanterre

\section{Édition imprimée}

Date de publication : 1 décembre 1999

Pagination : $31-54$

ISSN : 0246-8743

\section{Référence électronique}

Nathalie Vallée, Louis-Jean Boë et Muriel Stefanuto, « Typologies phonologiques et tendances

universelles. Approche substantialiste », Linx [En ligne], 11 | 1999, mis en ligne le 03 juillet 2012, consulté le 19 avril 2019. URL : http://journals.openedition.org/linx/863 ; DOI : 10.4000/linx.863

Ce document a été généré automatiquement le 19 avril 2019

Département de Sciences du langage, Université Paris Ouest 


\title{
Typologies phonologiques et tendances universelles. Approche substantialiste
}

\author{
Nathalie Vallée, Louis-Jean Boë et Muriel Stefanuto
}

\section{De l'intérêt des typologies}

1 La taxinomie vise à proposer un ordre à l'intérieur duquel seront classés les éléments d'un ensemble. C'est un des programmes fondateurs de toute science. Les botanistes, les zoologistes ont été peut-être les premiers spécialistes de ces grandes opérations de structuration. Avec de tels travaux se posent toute une série de questions de méthode. Où commence la variété? Quelles sont les limites d'une espèce ? Sur quels critères doit-on rassembler les éléments d'une même famille ? Au-delà de la complexité et de la diversité des éléments il faut savoir trouver la ressemblance, les traces d'une même organisation: maîtriser les différences, se détacher des caractères extérieurs pour accéder de plus en plus aux structures de moins en moins factuelles.

2 La typologie linguistique est actuellement en pleine maturation mais nous sommes encore loin du stade qu'avait atteint la botanique au $\mathrm{XVII}^{\mathrm{e}}$ siècle probablement parce que les problèmes fondamentaux de taxinomie affluent dès l'élaboration des bases de données représentatives des langues du monde. Il existe un très grand nombre de langues parlées à la surface du globe (entre 5000 et 8000 selon les sources), dont la plupart sont encore peu ou mal connues: on ne dispose pas de leurs descriptions systématiques à quel que niveau que ce soit (phonétique, phonologique, morphologique, lexical, syntaxique, sémantique... ). De plus, selon les sources, une même langue peut avoir des dénominations différentes. Il n'existe pas non plus de critères irréfutables permettant de faire la différence entre ce que l'on peut considérer comme une langue, un dialecte, un parler. Dans l'état actuel des recherches, on est encore loin de disposer d'un vaste échantillon des descriptions linguistiques pour les langues du monde (si la notion d'échantillon a ici un sens), alors que nombre d'entre elles sont en voie de disparition rapide (Ladefoged, 
1995), et avec elles, des éléments fondamentaux de la diversité linguistique de notre espèce (Ladefoged \& Maddieson, 1996).

3 Les études typologiques des structures sonores ont commencé à la fin du XIX siècle avec les travaux de Baudouin de Courtenay (1894), mais c'est Troubetzkoy, une figure de proue du Cercle Linguistique de Prague, qui ouvre l'ère des taxinomies phonologiques en 1939. Il écrit :

«J'ai mis au net tous les systèmes vocaliques que je connaissais par cœur (34 en tout) et j'ai essayé de les comparer les uns aux autres [...]. Les résultats sont extrêmement curieux [...]. Tous les systèmes se réduisent à un petit nombre de type et peuvent être représentés par un schéma symétrique [...]. Plusieurs lois "de la formation des systèmes" se laissent dégager sans peine [...]. Je crois que les lois empiriques acquises ainsi seront d'une grande importance, particulièrement pour l'histoire de la langue et sa reconstruction [...]. Elles devront être applicables à toutes les langues, aussi bien aux langues mères (Ursprachen) reconstruites théoriquement qu'aux divers stades de développement des langues historiquement attestées. » (note du 19-IX-1928)

列 du langage (Jakobson, 1941) et la définition des traits phonétiques (Hockett, 1955). Par la suite, les travaux les plus marquants vont s'effectuer aux USA dans une démarche descriptive associée à une recherche sur les universaux. Après le travail d'archivage publié dans l'International Journal of American Linguistics depuis 1944, et celui des missionnaires formés par le Summer Institute of Linguistics, la quête des universaux est marquée par :

- les travaux de Greenberg dans les années 1950 et la Conference on Language Universals à New-York en 1961;

- la discussion des propositions de Chomsky au Symposium on Universals in Linguistic Theory à Austin, en 1967.

Ces deux événements ont fortement contribué à faire reconnaitre le caractère institutionnel de ce champ de recherche avec pour objectifs la mise en évidence des structures de base communes à toutes les langues actuellement connues et la description de leur évolution. The Language Universal Project (1967-1976) a permis la constitution des Stanford Phonology Archives (Greenberg, Ferguson et Moravcsik, 1978) à partir desquelles sont nés des travaux d'importance concernant les classifications typologiques et l'étude des universaux phonologiques (Sedlack, 1969; Crothers, 1978; Maddieson, 1986; Vallée, 1994; Schwartz \& al, 1997a). Depuis Troubetzkoy, les données ont sans cesse été complétées et améliorées. Les interrogations sur le matériau abondent et diffèrent selon les auteurs. Toutefois les travaux typologiques ont davantage porté sur les systèmes vocaliques. Cet état de fait est probablement à relier au nombre plus important de consonnes dans les systèmes et à la multiplicité des paramètres de classement, rendant plus ardue l'émergence des types. Citons parmi les taxinomies consonantiques les travaux de (Hockett, 1955 ; Hagège, 1982 ; Maddieson, 1986 ; Lindblom \& Maddieson, 1988 ; Laver, 1994 ; Stefanuto, 1996 ; Vallée, 1998).

6 La plupart des études typologiques permettent parfois d'observer une association entre certains traits phonologiques et certaines aires géographiques (comme c'est le cas par exemple pour les langues du continent africain). Cependant, les types phonologiques et la grande majorité des tendances structurelles mises en évidence à partir d'inventaires représentatifs des langues du monde s'étendent au-delà des parentés génétiques et jusqu'à l'ensemble des familles linguistiques. Face à ce constat, les typologies des 
structures sonores ont pris une dimension particulière dans l'élaboration des théories phonologiques à partir des années 1970, avec les travaux en modélisation de Lindblom pour expliquer la structure des systèmes vocaliques à partir des contraintes de production et de perception de parole : les grandes tendances des systèmes sonores ne seraient-elles pas, pour une part, le résultat de contraintes articulatoires et/ou perceptives? Ne serait-ce pas la substance qui structure la forme plutôt que l'inverse? (Liljencrants \& Lindblom, 1972). Ces questions conduisent à remettre en perspective les relations traditionnelles d'indépendance entre forme et substance (Vallée, 1994:12-31; Boë, 1997). C'est dans cette voie de recherche que nous avons choisi d'élaborer des typologies approfondies des structures phonologiques, avec mise en évidence des tendances générales. Après une présentation détaillée des données, dans un premier temps, nous proposons une typologie à partir de laquelle sont mises au jour les grandes tendances des systèmes consonantiques. Rapprochées de données disponibles sur l'acquisition du langage, elles devraient permettre, dans une perspective proche, de tester des paradigmes d'émergence des structures sonores: parmi les différentes étapes de l'ontogenèse, celle du babillage apparaît comme fondamentale puisqu'elle marque les "précurseurs» des attributs universels du langage, au sens défini par (MacNeilage \& Davis, 1990). Dans un deuxième temps, nous présentons des données typologiques et tendances générales des systèmes vocaliques des langues du monde que nous exploitons comme données d'entrée pour le modèle ICP de prédiction des structures phonologiques (Schwartz \& al. 1997b). Basé sur la Théorie de la Dispersion-Focalisation (TDF), ce modèle intègre des propriétés des mécanismes de production et de perception des sons du langage.

7 Nous avons donc choisi d'élaborer et d'explorer des typologies phonologiques avec un cadre épistémologique dans lequel la faiblesse intrinsèque de la seule démarche inductive - pouvant entraîner l'erreur d'affirmer le conséquent d'observations partielles - est compensée par des principes et des données externes au langage du point de vue formel, c'est-à-dire indépendantes des contraintes purement cognitives du langage.

\section{Les données}

La constitution d'un échantillon représentatif de l'inventaire phonologique des langues du monde, dans leur état actuel (synchronie), est devenu un outil indispensable pour examiner le contenu et la structure des systèmes sonores des langues rencontrées à la surface du globe. L'essor des techniques numériques a favorisé la constitution de banques de données de plus en plus importantes pour faciliter ce champ de recherche. Ce n'est que depuis une quinzaine d'années que l'on dispose de bases de données phonologiques « représentatives » des langues du monde. Constituées à partir des archives de Stanford, les bases UPSID (UCLA Phonological Segment Inventory Database), dont la première version publiée contient 317 systèmes phonologiques (Maddieson, 1986), et la plus récente, 451 langues répertoriées (Maddieson \& Precoda, 1990), contiennent des données phonologiques génétiquement pondérées et descriptivement harmonisées (Maddieson, 1986 : 5-6). Nous les avons implantées à l'ICP pour nourrir les recherches typologiques sur les consonnes et les voyelles.

UPSID se veut être un échantillon représentatif d'un point de vue génétique et géographique d'au moins $5 \%$ de l'ensemble des langues du monde. Dix-huit familles de langues (Table 1), réparties sur les cinq continents, y sont représentées. Est inclus dans 
l'inventaire au moins un dialecte par groupe de langues (sous-famille) sur la base d'une distance génétique de séparation des langues d'au moins 1500 ans, " a long enough period for substantial independant developments to occur in the phonological patterns of any two languages belonging to the same larger family." (Maddieson, 1991a: 348). C'est une marge d'assurance plus large que celle estimée par (Ruhlen, 1987:6) : « under most circumstances 500 years is probably sufficient to seriously impair mutual intelligibility, and 1000 years will usually obliterate it entirely. »Cette distance de 1500 ans correspond à la séparation entres langues germaniques de la branche nordique (islandais, danois, suédois, norvégien) et langues germaniques de la branche occidentale (allemand, néerlandais, anglais, frison, anglo-frison), pour lesquelles on estime un certain degré d'indépendance: entre les langues issues d'une même souche la distance génétique, bien qu'elle reste difficile à apprécier, est d'autant plus grande que leur séparation est plus ancienne.

Le nombre de locuteurs ne rentre en aucune manière dans la sélection des langues de l'échantillon : "The size of extent populations of speakers of languages is an accident of political and social history that is quite irrelevant to the questions relating to the structure of human languages "(Maddieson, 1986 : 158).

11 Comme nous l'avons vu, Troubetzkoy avait fait appel, de mémoire, à 34 langues, Sedlak (1969) a travaillé sur 150 langues tirées des Stanford Phonology Archives et Crothers (1978) sur 209. UPSID, enrichie à 451 langues, est actuellement une des rares sources d'étude des systèmes phonologiques des langues du monde qui permette de dresser un inventaire des types de systèmes et de repérer les objets fondamentaux porteurs d'informations sur les sons du langage humain et sur la formation des systèmes sonores. Pour reprendre le propos de Maddieson (1991b), UPSID doit être considérée comme une fenêtre par laquelle il est possible d'entrevoir un état actuel des langues du monde.

$\mathrm{Au}$ niveau de la répartition par famille, la première version d'UPSID présente 17 familles de langues plus trois langues isolées : basque, bourouchaski, aïnou alors que la deuxième comporte 18 familles et quatre langues isolées qui sont nivkh (paléo-sibérien), basque, bourouchaski et kète. Dans la deuxième version, sont constatés également le glissement de la famille indo-pacifique dans le phylum papou, l'intégration de l'aïnou dans la famille de langue ouralo-altaïque, le kète comme langue isolée et non plus rattachée à la famille paléo-sibérienne, le tchouktchi-kamchatkan comme une famille à part entière (3 langues : tchouktchi, itelmen, koryak) dans UPSID $_{451}$, tout comme le na-déné, intégré dans la famille amérindienne dans UPSID $_{317}$ et qui figure comme famille dans la version plus récente représentée par les langues : eyak, hupa, chipewyan, navajo, haïda, ahtna et tlingit. Le passage d'UPSID ${ }_{317}$ à UPSID ${ }_{451}$ est bien plus qu'une simple addition: 192 langues ont été ajoutées et 58 autres ôtées d'UPSID ${ }_{317}$. Une partie des langues conservées a été modifiée (prise en compte de nouvelles références). Ces changements relèvent de deux types : le premier porte sur le nombre de phonèmes (exemple, 47 systèmes ont vu soit baisser, soit augmenter leur nombre de voyelles), le deuxième correspond à une différence de transcription API des unités du système. Malgré un certain nombre de modifications, l'analyse typologique d'UPSID ${ }_{451}$ (Abbadeni, 1996; Alcantara, 1998) n'apporte pas de changements fondamentaux dans les grandes tendances des systèmes phonologiques mises en évidence par Maddieson (1986), Vallée (1994), Stefanuto (1996). 
Table 1. Les langues d'UPSID ${ }_{451}$ classées par famille

\begin{tabular}{|c|c|}
\hline $\begin{array}{l}\text { Famille } \\
\text { (Nombre de } \\
\text { langues) }\end{array}$ & Langues \\
\hline $\begin{array}{l}\text { indo- } \\
\text { européen } \\
(23)\end{array}$ & $\begin{array}{l}\text { grec, irlandais, breton, allemand, norvégien, lithuanien, russe, bulgare, français, } \\
\text { espagnol, roumain, farsi, pashto, kurde, hindi-urdu, bengalais, cachemirien, } \\
\text { cinghalais, albanais, arménien, népalais, konkani, ormuri }\end{array}$ \\
\hline $\begin{array}{l}\text { ouralo- } \\
\text { altaïque (28) }\end{array}$ & $\begin{array}{l}\text { aïnou, khanty, mari, komi, finnois, hongrois, saami, nenets, nganasan, turc, } \\
\text { azerbaïdjanais, chouvache, yakoute, kirghiz, bashkir, selkup, tuva, khalkha, } \\
\text { even, nanai, mandchou, coréen, japonais, youkaghir, monguor, moghol, ouzbek, } \\
\text { dagur }\end{array}$ \\
\hline $\begin{array}{l}\text { austro- } \\
\text { asiatique } \\
(14)\end{array}$ & $\begin{array}{l}\text { mundari, kharia, khasi, vietnamien, sedang, cambodgien, parauk, sre, brao, } \\
\text { khmu?, nicobarais, pacoh, kur, bruu }\end{array}$ \\
\hline $\begin{array}{l}\text { austro-thaï } \\
(39)\end{array}$ & $\begin{array}{l}\text { thaïlandais, lakkia, yay, soui, kam, po-ai, lungchou, atayal, javanais, malgache, } \\
\text { cham, sama, batak, tagalog, sa'ban, chamorro, rukai, tsou, adzera, roro, kaliai, } \\
\text { iai, hawaiien, tigak, lenakel, lue, ivatan, mor, pohnpeien, tiruray, lai, kwaio, } \\
\text { paiwan, iban, gelao, tetun, fidjien, irarutu, maranao }\end{array}$ \\
\hline $\begin{array}{l}\text { sino-tibétain } \\
(21)\end{array}$ & $\begin{array}{l}\text { mandarin, taishan, hakka, changzhou, xiamen, fuzhou, bai, tamang, dafla, } \\
\text { burmese, lahu, jingpho, ao, chin, bodo, karen, mien, newari, hmong, phlong, naxi }\end{array}$ \\
\hline $\begin{array}{l}\text { caucasien } \\
(7)\end{array}$ & géorgien, kabardien, lak, rutul, bats, archi, avar \\
\hline $\begin{array}{l}\text { autres } \\
\text { familles } \\
\text { euro- } \\
\text { asiatiques } \\
\text { (7) }\end{array}$ & nivkh, kète, tchouktchi, koryak, itelmen, basque, bourouchaski \\
\hline dravidien (6) & telugu, kota, kouroukh, koya, tulu, brahoui \\
\hline $\begin{array}{l}\text { niger-congo } \\
(55)\end{array}$ & $\begin{array}{l}\text { moro, kadugli, kpelle, bisa, bambara, dan, wolof, diola, temne, dagbani, senadi, } \\
\text { tampulma, bariba, ewe, akan, igbo, ga, lelemi, efik, birom, tarok, amo, beembe, } \\
\text { ogbia, ejagham, zoulou, teke, doayo, gbeya, azande, aizi, mumuye, klao, aghem, } \\
\text { kpan, kohumono, yorouba, bobo-fing, noni, gwari, ewondo, jomang, sango, bete, } \\
\text { konyagi, mbum, isoko, fe ?fe ?, ndut, ijo, lua, alladian, mambila, mba-ne, dogon }\end{array}$ \\
\hline $\begin{array}{l}\text { nilo- } \\
\text { saharien } \\
(23)\end{array}$ & $\begin{array}{l}\text { songhaï, kanouri, maba, fur, maasai, luo, nubien, nyangi, ik, sebei, tama, temein, } \\
\text { nera, tabi, mursi, lugbara, yulu, berta, kunama, koma, daju, dinka, nyimang }\end{array}$ \\
\hline $\begin{array}{l}\text { afro- } \\
\text { asiatique } \\
(26)\end{array}$ & $\begin{array}{l}\text { arabe, tigre, amharique, socotri, néo-araméen, chleuh, tamasheq, somali, awiya, } \\
\text { iraqw, beja, kullo, dizi, kefa, hamer, hausa, angas, margi, ngizim, kanakuru, kera, } \\
\text { dahalo, kotoko, dangaleat, tera, lame }\end{array}$ \\
\hline
\end{tabular}




\begin{tabular}{|c|c|}
\hline khoisan (4) & hadza, sandawe, !xu, nama \\
\hline na-déné (7) & haida, tlingit, navajo, hupa, chipewyan, ahtna, eyak \\
\hline $\begin{array}{l}\text { Amérique } \\
\text { du Nord (58) }\end{array}$ & $\begin{array}{l}\text { percé, klamath, maidu, wintu, zoque, tzeltal, totonac, k'ekchi, mixe, huave, } \\
\text { mazahua, mazatec, mixtec, chatino, tseshaht, kwakw'ala, quileute, lushootseed, } \\
\text { papago, luiseno, hopi, yaqui, picuris, karok, pomo, diegueno, achumawi, yana, } \\
\text { shasta, tol, zuni, acoma, ojibwa, tonkawa, wiyot, seneca, wichita, dakota, yuchi, } \\
\text { tunica, alabama, wappo, nahuatl, kawaiisu, amuzgo, bella coola, tlapanec, } \\
\text { chehalis, tsimshian, caddo, huasteco, yucatec, shuswap, miwok, jacaltec, } \\
\text { cherokee, kiowa, chinantec }\end{array}$ \\
\hline $\begin{array}{l}\text { Amérique } \\
\text { du Sud (66) }\end{array}$ & $\begin{array}{l}\text { itonama, bribri, pirahã, cayapa, paez, ocaina, muinane, caraïbe (galibi), } \\
\text { kaingang, apinaye, amahuaca, epena pedee, tacana, axluxlay, abipon, } \\
\text { nambiquara, arabela, auca, quechua, jaqaru, tehuelche, wapishana, caraïbe } \\
\text { insulaire, amuesha, campa, guajiro, moxo, guarani, siriono, guahibo, ticuna, } \\
\text { barasano, siona, jivaro, cofan, mapudungu, resigaro, yagua, cayuvava, huari, } \\
\text { hixkaryana, yucuna, jebero, iranxe, cubeo, tarascan, japreria, panare, bororo, } \\
\text { andoke, warao, akawaio, paya, shiriana, bakairi, cuna, maxakali, qawasqar, } \\
\text { movima, saliba, guambiano, camsa, cacua, trumai, ache, iate }\end{array}$ \\
\hline $\begin{array}{l}\text { eskimo- } \\
\text { aléoute (3) }\end{array}$ & aléoute, inuit, yupik \\
\hline $\begin{array}{l}\text { australien } \\
(25)\end{array}$ & $\begin{array}{l}\text { garawa, yanyuwa, waray, murinhpatha, maung, tiwi, burarra, nunggubuyu, } \\
\text { alawa, malakmalak, bardi, wik-munkan, désert occidental, arrernte, gugu- } \\
\text { yalanji, ya, diyari, bandjalang, kalkatungu, yidiny, dyirbal, ngiyambaa, } \\
\text { mbabaram, ngarinjin, yolngu }\end{array}$ \\
\hline papou (39) & $\begin{array}{l}\text { andamanese, asmat, kwoma, sentani, nimboran, iwam, selepet, gadsup, yagaria, } \\
\text { kewa, chuave, dani, wantoat, dadibi, fasu, suena, dera, kunimaipa, yareba, koiari, } \\
\text { taoripi, nasioi, rotokas, nambakaengo, angaatiha, wahgi, yawa, usan, baining, } \\
\text { yessan-mayo, woisika, alamblak, amele, kiwai, waris, vanimo, savosavo, makian, } \\
\text { ekari }\end{array}$ \\
\hline
\end{tabular}

\section{Systèmes consonantiques : des tendances universelles à l'ontogenèse}

\section{1 Éléments de taxinomie}

13 La classification traditionnelle des consonnes repose sur les lieux et les modes d'articulation. Ces paramètres constituent bien évidemment les dimensions élémentaires de notre étude typologique. UPSID $_{451}$ répertorie 920 phonèmes dont 654 segments consonantiques, répartis sur 13 lieux d'articulation (Figure 1) et une quinzaine de modes. On dénombre 153 plosives, 61 implosives, occlusives éjectives et clicks non affriqués, 134 fricatives, fricatives éjectives, 155 affriquées, affriquées éjectives et clicks affriqués, 95 vibrantes, battues et approximantes centrales et latérales, 51 nasales et 5 consonnes de type $h$ sounds. 
Figure 1. Lieux d'articulation consonantiques (UPSID451)

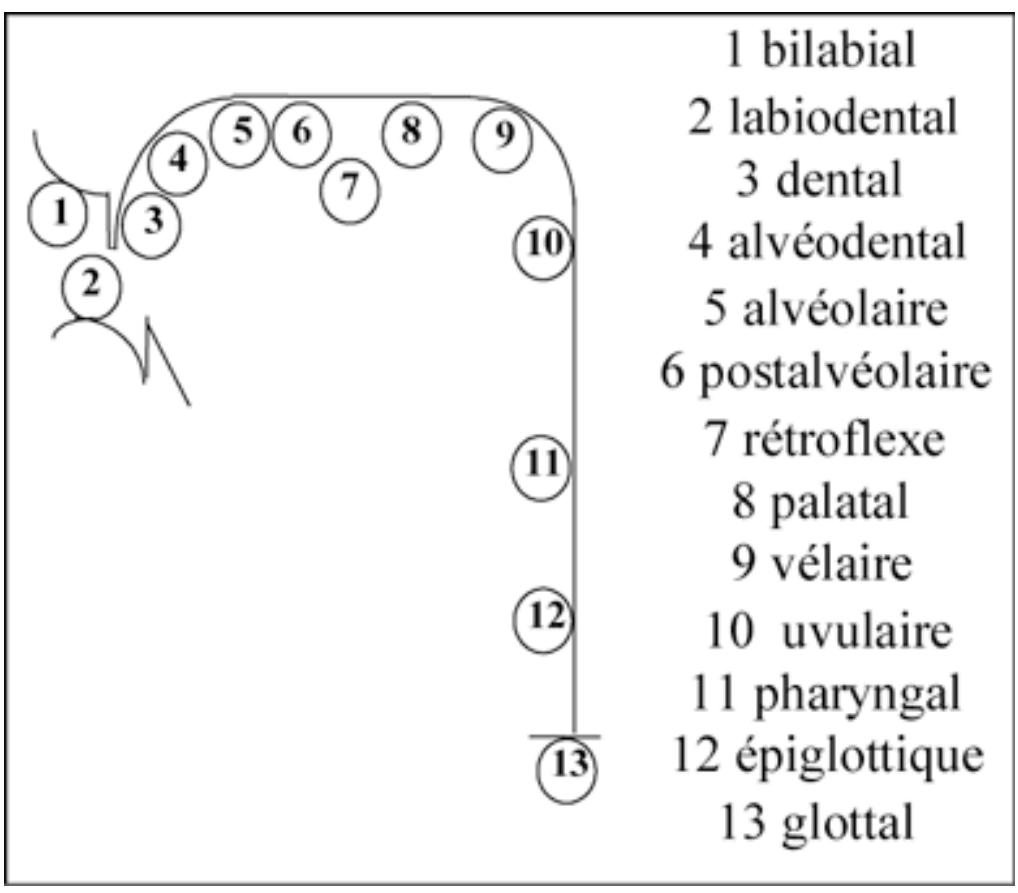

Les modes ont été regroupés sous 7 catégories classant ainsi les consonnes en plosives (dont implosives, éjectives, occlusives glottales), nasales, fricatives (dont celles éjectives et $h$ sounds), affriquées (dont éjectives), approximantes, vibrantes/battues, clicks.

Nous avons élaboré notre typologie avec une détermination des lieux plus détaillée que celle de (Maddieson, 1986) en composant avec celles proposées par Creissels (1994) et Ladefoged \& Maddieson (1996). Bien que désignée par un mode, nous avons conservé la classe des rétroflexes comme le proposent Maddieson (1986), Ladefoged \& Maddieson (1996) et l'API de 1996. Une répartition de cette catégorie de consonnes, qui recouvrent plusieurs lieux (alvéodental, alvéolaire, prépalatal...), nécessiterait une investigation des sources. Soulignons que la comparaison avec les taxinomies antérieures n'est toutefois pas aisée car toutes diffèrent dans la préparation des données et les méthodes de classement.

\subsection{Distributions}

Les systèmes phonologiques ont le plus souvent entre 18 et 25 consonnes, avec un mode à 22: minimum 6 pour le rotokas (langue indo-pacifique, 11 phonèmes) et maximum 95 (dont 48 clicks) pour le !xũ (famille khoisan, 141 phonèmes). La moyenne par langue est de 22 consonnes dont 7.8 plosives, 4.1 fricatives, 3.3 nasales, 2.9 approximantes, 2 affriquées, 0.6 éjective, 0.52 vibrante ou battue, 0.25 implosive, 0.2 click.

La Table 2 donne la répartition des consonnes (654 au total) par lieux d'articulation. Les plus fréquentes, tous modes confondus, sont les alvéodentales (15.3\%), suivies des bilabiales (14.3\%) puis des vélaires (12.6\%). Le regroupement des consonnes dentales, alvéodentales, alvéolaires, postalvéolaires et de l'ensemble des rétroflexes en une catégorie coronale permet de mieux apprécier l'écrasante proportion de celle-ci : $44.5 \%$ dans UPSID $_{317}$. Bien que cette catégorie regroupe une grande diversité d'articulations 
(Ladefoged \& Maddieson, 1996), ce choix est tout à fait acceptable pour l'élaboration de notre typologie. En effet, le trait de lieu ne sert que rarement à distinguer phonologiquement des consonnes de ce type, sauf pour les affriquées, et en tout cas pas entre coronales antérieures (Keating, 1990).

La répartition des consonnes d'UPSID ${ }_{317}$ par mode montre que certains sont plus exploités que d'autres: plosives $38.6 \%$, fricatives $20.2 \%$, nasales $14.6 \%$, approximantes $13 \%$, affriquées $9.6 \%$, vibrantes ou battues $3.9 \%$. Parmi les plus fréquentes arrivent en tête les occlusives coronales (type $/ \mathrm{t} /$ ), orales et sourdes $(97.5 \%)$, les nasales bilabiales et coronales (types $/ \mathrm{m} /$ et $/ \mathrm{n} /$, présentes aussi dans plus de 9 langues sur 10), suivies de $/ \mathrm{k} /$, $/ \mathrm{j} /, / \mathrm{p} /$ rencontrées dans plus de $80 \%$ des langues, /w/ et $/ \mathrm{s} /$ dans 2 langues sur $3, / \mathrm{d} /$, / $\mathrm{b} /, / \mathrm{h} /$, dans plus de $60 \%, / \mathrm{l} /, / \mathrm{g} /, / \mathrm{y} /, / \mathrm{l} /$ dans la moitié des langues.

Table 2. Fréquences d'occurrences des lieux d'articulation consonantiques (UPSID $\left.{ }_{317}\right)$

\begin{tabular}{|l|l|}
\hline alvéodental & $15.3 \%$ \\
\hline bilabial & $14.3 \%$ \\
\hline vélaire & $12.6 \%$ \\
\hline palatal & $10 \%$ \\
\hline \hline apico-alvéolaire & $9.9 \%$ \\
\hline glottal & $7.6 \%$ \\
\hline lamino-postalvéolaire & $6.7 \%$ \\
\hline labiovélaire & $5.1 \%$ \\
\hline dental & $5 \%$ \\
\hline lamino-alvéolaire & $3.4 \%$ \\
\hline labiodental & $3.3 \%$ \\
\hline rétroflexe & $2.9 \%$ \\
\hline uvulaire & $1.7 \%$ \\
\hline autres & $<1 \%$ \\
\hline
\end{tabular}




\subsection{Systèmes}

\subsubsection{Taille des systèmes de lieux et modes d'articulation}

L'élaboration d'une typologie par lieux et modes des systèmes consonantiques fait apparaitre une forte corrélation entre la taille des systèmes de lieux et les différents modes d'articulation (Table 3).

- Dans la plupart des langues, les plosives et les nasales mettent en jeu respectivement de 3 à 6 , et de 1 à 6 lieux. Le mode plosif est le seul universellement exploité par les systèmes sonores. Il est aussi le seul à utiliser systématiquement les oppositions de lieux : les langues en distinguent au moins trois.

- Les fricatives et les approximantes constituent les catégories qui recrutent le plus grand nombre d'oppositions de lieux : le mode fricatif est beaucoup plus largement distribué entre les différentes tailles de systèmes. Si le jeu des oppositions de lieux des fricatives est souvent plus complexe que celui des plosives, il est important de préciser qu'il n'est pas exploité par $8 \%$ des langues de l'échantillon - alors que toutes possèdent des plosives.

- Dans la catégorie des vibrantes et battues (trills, flaps et taps), les systèmes ne présentent que rarement une opposition de lieu, et en aucun cas ils n'en distinguent plus de 2. Cette même tendance se retrouve pour les affriquées.

\section{Plus généralement :}

- Les langues exploitent systématiquement l'opposition de lieu pour les consonnes plosives ; près de la moitié les répartissent dans des systèmes à 4 lieux.

- L'opposition de lieu est rare pour les affriquées, exceptionnelle pour les vibrantes/battues.

- Les approximantes sont de préférence réparties sur 3 lieux d'articulation.

- Les fricatives se répartissent plus largement dans diverses tailles de systèmes de lieux. 
Table 3. Taille des systèmes de lieux et leur représentativité dans UPSID $_{317}$

\begin{tabular}{|l|l|l|l|l|l|l|l|l|}
\hline & 1 lieu & $\begin{array}{l}2 \\
\text { lieux }\end{array}$ & $\begin{array}{l}3 \\
\text { lieux }\end{array}$ & $\begin{array}{l}4 \\
\text { lieux }\end{array}$ & $\begin{array}{l}5 \\
\text { lieux }\end{array}$ & $\begin{array}{l}6 \\
\text { lieux }\end{array}$ & $\begin{array}{l}7 \\
\text { lieux }\end{array}$ & $\begin{array}{l}8 \\
\text { lieux }\end{array}$ \\
\hline trills, flaps, taps & $67.2 \%$ & $4.7 \%$ & & & & & & \\
\hline affriquées & $44.8 \%$ & $20.8 \%$ & $6.9 \%$ & $0.3 \%$ & & & & \\
\hline plosives & & & $28.4 \%$ & $43.2 \%$ & $22.4 \%$ & $5.9 \%$ & & \\
\hline nasales & $2.5 \%$ & $31.5 \%$ & $30.6 \%$ & $25.9 \%$ & $4.5 \%$ & $2.2 \%$ & & \\
\hline approximantes & $8.2 \%$ & $26.5 \%$ & $49.8 \%$ & $8.2 \%$ & $2.5 \%$ & $0.3 \%$ & $0.3 \%$ & \\
\hline fricatives & $3.4 \%$ & $18.3 \%$ & $25.9 \%$ & $21.1 \%$ & $14.2 \%$ & $7.3 \%$ & $1.9 \%$ & $0.9 \%$ \\
\hline nombre d'oppositions de & 0 & 1 & 3 & 6 & 10 & 15 & 21 & 28 \\
\hline lieux & & & & & & & \\
\hline
\end{tabular}

Le nombre d'oppositions de lieux varie selon les modes ; de 0 (c'est-à-dire 1 lieu par système) à 28 (8 lieux).

Les valeurs présentées ne tiennent pas compte du trait de voisement : $28.4 \%$ des 317 langues ont un système de plosives à 3 lieux,

43.2\% possèdent des plosives réparties sur 4 lieux, etc.

\subsubsection{Systèmes de lieux par modes}

21 La Table 4 rend compte du contenu des systèmes de lieux par mode d'articulation. Sont représentées les répartitions des plosives, nasales, fricatives et affriquées par nombre de lieux d'articulation.

22 Il n'existe pas de langues sans plosives, ni même n'en ayant qu'une seule. Lorsqu'un système les répartit sur 2 lieux, l'un est coronal, l'autre bilabial ; sur 3 lieux : coronal, bilabial et vélaire. C'est à 4 qu'apparaît le lieu glottal; si le système répartit ses consonnes entre 5 lieux d'articulation, il recrute les régions bilabiale, coronale, vélaire, uvulaire et glottale.

23 S'il y a une seule consonne nasale dans un système, elle est de type coronal. S'il en possède 2, elles sont de type bilabial et coronal. Réparties sur 3 lieux, s'ajoute une nasale vélaire. Puis à 4 , apparaît le lieu palatal.

24 Si les fricatives d'un système n'exploitent qu'un lieu d'articulation, il s'agit de la région alvéolaire donc d'une coronale ; 2 lieux : alvéolaire et glottal ; à 3 lieux apparaissent les fricatives labiodentales; à 4 est exploitée la région palatale et à 5 lieux apparaissent les fricatives vélaires.

25 Les affriquées, quant à elles, se répartissent dans les régions d'articulation des coronales, quelle que soit la taille du système de lieu (1 à 3). 
De la répartition des systèmes de lieux par catégories nous retenons les tendances suivantes :

- Comme pour les systèmes vocaliques (Vallée, 1994), le système de lieux à n éléments contient le système à n-1 lieux consonantiques.

- Lorsqu'il n'y a qu'un lieu, quelle que soit la catégorie (plosives, fricatives ou autres), il s'agit de consonnes coronales (lieux 4 et 5 ).

- 95\% des langues contrastent sur 3 lieux à l'intérieur des catégories (plus d'une langue sur 3 pour les nasales).

- Une taille de système domine chaque catégorie : $43 \%$ des langues ont 4 lieux pour les plosives et $74 \%$ ont 3 ou 4 lieux) ; 32\% des langues répartissent leur nasales sur 3 lieux (59\% sur 3 et 4 ) ; $28 \%$ présentent 3 lieux pour les fricatives ( $48 \%$ sur 3 et 4 ) ; 43\% n'ont qu'un lieu d'articulation pour les affriquées.

- Les combinaisons à 5 lieux sont présentes dans seulement 36\% des langues, essentiellement dans les plosives orales (97 langues).

- Les combinaisons à 6, 7 ou 8 lieux sont peu répandues (15\% des langues). On les rencontre essentiellement dans les systèmes de fricatives.

Table 4. Les catégories consonantiques (plosives, nasales, fricatives, affriquées) d'UPSID ${ }_{451}$ ' classées par nombre de lieux d'articulation avec le pourcentage de langues correspondant (par exemple, $31 \%$ des 451 langues présentent un système de plosives réparties sur 3 lieux, $43 \%$ sur 4 lieux etc. ; 0 lieu correspondant à une absence de catégorie). Ne sont portés dans cette table que les systèmes les plus répandus.

\begin{tabular}{|l|l|l|l|l|l|l|l|l|}
\hline lieux & \multicolumn{2}{l}{ plosives } & \multicolumn{2}{l|}{ nasales } & \multicolumn{2}{l|}{ fricatives } & \multicolumn{2}{l|}{ affriquées } \\
\hline 0 & $0 \%$ & - & $3.5 \%$ & - & $7 \%$ & - & $33 \%$ & - \\
\hline 1 & $0 \%$ & - & $1.8 \%$ & coronal & $6.6 \%$ & coronal & $42 \%$ & coronal \\
\hline 2 & $0.2 \%$ & & $31 \%$ & & $18 \%$ & & $20 \%$ & 2 \\
\hline 3 & $31 \%$ & coronvélaire & $32 \%$ & vélaire & $28 \%$ & labiodental & $4.9 \%$ & onal 3 \\
\hline 4 & $43 \%$ & glottal & $27 \%$ & palatal & $20 \%$ & coronal 2 & - & - \\
\hline 5 & $21 \%$ & & $3.5 \%$ & & $11 \%$ & & - & - \\
\hline
\end{tabular}

Nos résultats confirment que les plosives sont de loin les phonèmes consonantiques "vedettes ", omniprésentes dans les langues et que, comptabilisées avec les nasales pour chaque langue, leur nombre est beaucoup plus important que le nombre de fricatives (figure 2), à une douzaine d'exceptions près. 
Figure 2. Nombre de fricatives en fonction du nombre d'occlusives dans UPSID ${ }_{317}$.

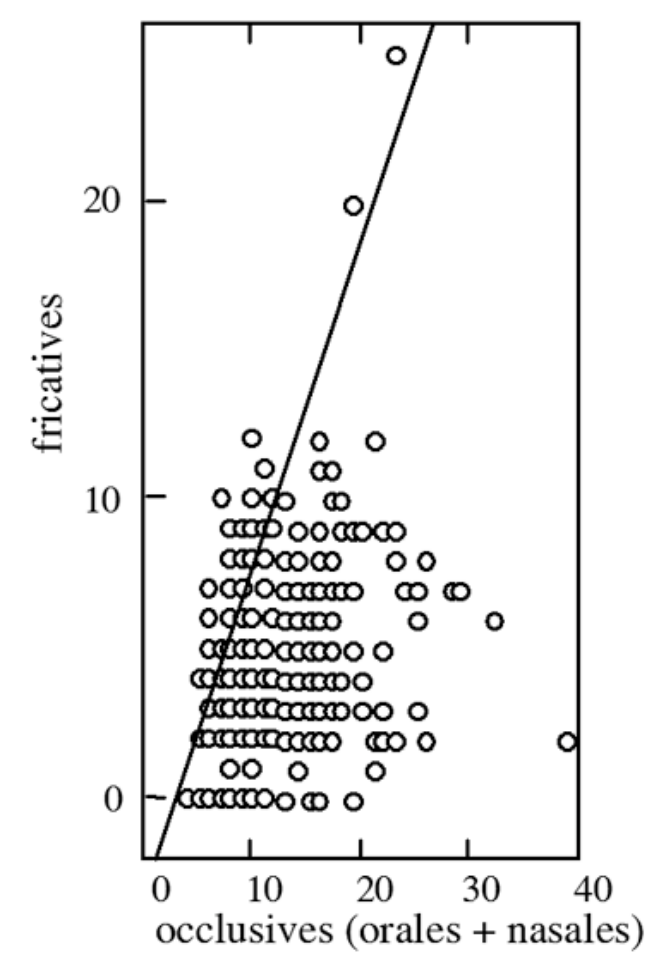

\subsubsection{Rapport sourdes/sonores}

Dans la catégorie des plosives orales, les sourdes dominent largement les sonores (64\%) : on relève $30 \%$ de sourdes en plus dans les bilabiales, $50 \%$ de sourdes en plus dans les coronales, $60 \%$ dans la catégorie vélaire. Font exception à cette tendance, les langues du continent africain - familles niger-kordofanienne, nilo-saharienne et afro-asiatique : elles présentent en moyenne 1,5 fois plus de plosives bilabiales sonores que de sourdes.

Ce rapport sourdes/sonores est encore plus écrasant pour les fricatives: les langues présentent 5 fois plus de /s/ que de /z/, 3,2 fois plus de $/ \mathrm{S} /$ que de $/ 3 /$ et presque 2 fois plus de /f/ que de /v/. Quel que soit le lieu d'articulation, le trait sourd concerne $72 \%$ des fricatives.

Les affriquées sont également majoritairement sourdes (74\%).

\subsection{Universaux consonantiques et ontogenèse}

31 Nous avons rassemblé les résultats de plusieurs études sur le contenu consonantique des productions à différents stades du babillage canonique (réduplication de syllabes identiques) ou panaché (avec variation des types de closions). L'ensemble de ces études couvre une période de 6-10 (Boysson-Bardies, 1996) à 15-24 mois (Stoel-Gammon, 1985) dans un environnement linguistique anglo-américain, français et de manière plus étendue sur 15 langues (Locke, 1983). (Boysson-Bardies) relève que [p b $\mathrm{m} \mathrm{t} \mathrm{d} \mathrm{n} \mathrm{k} \mathrm{g} \mathrm{p]}$ constituent $80 \%$ des productions « consonantiques » dans les premiers mois du babillage, [p b $\mathrm{m} \mathrm{t} \mathrm{d]} \mathrm{étant} \mathrm{les} \mathrm{plus} \mathrm{fréquentes.} \mathrm{La} \mathrm{revue} \mathrm{de} \mathrm{question} \mathrm{de} \mathrm{(Locke,} \mathrm{1983)} \mathrm{livre} \mathrm{par} \mathrm{ordre}$ décroissant de fréquence l'inventaire des consonnes représentant 95\% des occurrences 
produites par des enfants âgés de 6 à 15 mois : [b] et [m], [p], [d], [h] et [n], [t], [g] et [k], [j] et [w], [s]. Les observations de (Robb \& Bleile, 1994), sur une période plus longue (de 8 à 25 mois), montrent que chez les 8-12 mois plusieurs types de closions co-émergent. Les productions les plus fréquentes sont des plosives (orales et nasales), de lieu alvéolaire et bilabial. Ils confirment ainsi Davis \& MacNeilage (1994), qui relèvent qu'au cours des 7-12 mois, ces consonnes couvrent $84 \%$ des productions [t d] 46\%, [n] 18\%, [p b] 15\%, [m] 5\%); les vélaires et glottales sont plus tardives. Enfin, l'étude de (Stoel-Gammon, 1985) rend compte de l'apparition des segments consonantiques pour la période 15-24 mois : [b d m n jwh].

Si on met en parallèle ces résultats sur l'acquisition du langage avec les données typologiques sur les langues du monde, on constate que les types de closions produits dans les premiers mois du babillage (vers 8-12 mois), ainsi que les sons acquis plus tardivement au cours de la deuxième année, correspondent aux phonèmes consonantiques les plus fréquents dans les langues (l'ordre d'émergence mis à part). On remarque aussi que les systèmes de lieux préférés, mis en évidence par mode dans l'analyse typologique, sont dominants dans les productions du babillage : alvéolaire et bilabial, devant vélaire et glottal, pour le mode plosif (oral ou nasal) ; alvéolaire et glottal ([s] et [h]) pour le mode fricatif qui est moins fréquent ; labiovélaire, coronal et palatal pour les approximantes [w], [l], [j], acquises plus tardivement.

Ces constatations nous permettent alors de formuler l'hypothèse que les sons des langues sont sans doute puisés dans le stock des capacités potentielles du babillage. (Locke \& Pearson, 1992) n'hésitaient pas à conclure :

«Infants heavily favor stop consonants over fricatives, and there are languages that have stops and no fricatives but no languages that exemplify the reverse pattern.

[Such] "phonologically universal" patterns, which cut across languages and speakers are, in effect, the phonetic properties of Homo sapiens. » (p. 96)

L'ontogenèse peut ainsi contribuer à fournir, à l'évidence, des éléments déterminants sur les dépendances entre certaines tendances générales des systèmes phonologiques et les capacités sensori-motrices de l'Homo loquens

\section{Consonnes vs. voyelles}

Pour l'ensemble des langues la corrélation entre le nombre de consonnes et le nombre de voyelles n'est pas significative (Figure 3). Seule régularité, chaque langue possède plus de consonnes que de voyelles (à 2 exceptions près : le pawaian (famille austro-thaï) avec 12 voyelles et 10 consonnes; et l'apinaye (famille sud-amérindienne, groupe macro-ge), 17 voyelles et 13 consonnes). 
Figure 3. Nombre de voyelles en fonction du nombre de consonnes dans les systèmes d'UPSID ${ }_{317}$.

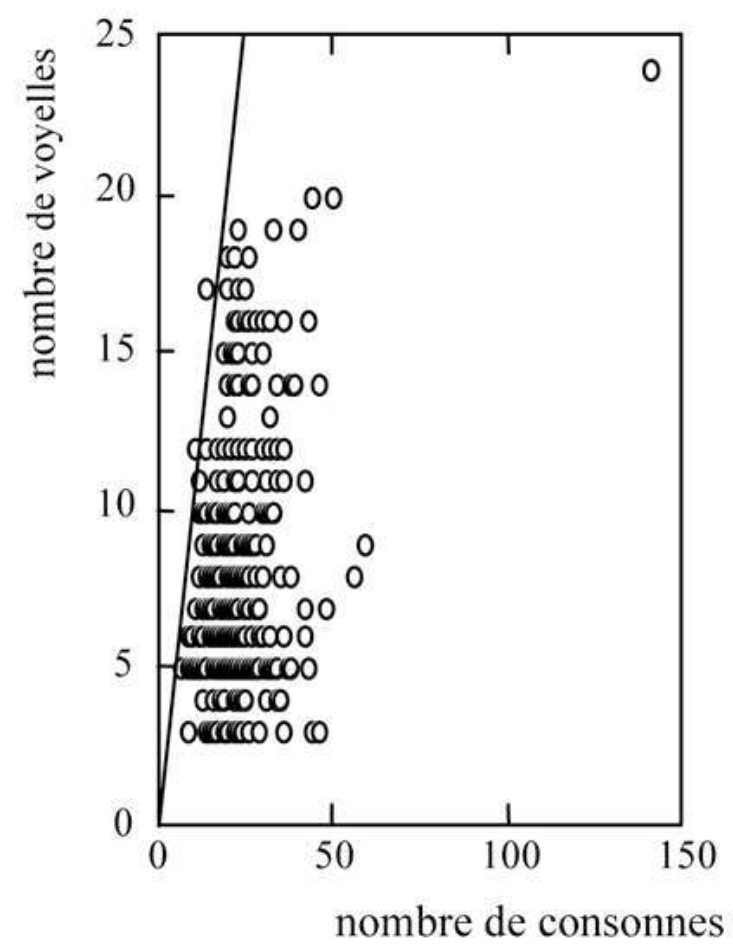

\section{Systèmes vocaliques : des tendances universelles à la prédiction}

\subsection{Des universaux vocaliques}

L'analyse menée à partir d'UPSID atteste que le potentiel linguistique, phonologique, des langues du monde puise dans un ensemble de 37 possibilités vocaliques et que parmi ces possibilités, les langues ne choisissent pas leurs unités distinctives sur des critères arbitraires.

Les systèmes vocaliques recrutent 3 à 28 phonèmes mais deux tiers d'entre eux ont entre 5 et 7 voyelles. La comparaison des systèmes les plus fréquents (tables $5 a$ \& $5 b$ ) met en évidence un ordre d'apparition des voyelles dans les systèmes. Les 3 «vedettes " / $a v /$ sont présentes dans $97 \%$ des langues. S'y ajoute la voyelle antérieure /'e'/ dans le système à 4 le mieux représenté. Le système à 5 /i'e'a'o'u/ est de loin le plus " populaire » dans les langues du monde. C'est le cas dans 3 des 4 grands groupes linguistiques (eurasien, américain, australien), alors que c'est le système périphérique à 7 /i e $\varepsilon$ a $0 \mathrm{o} u$ / qui est majoritaire en Afrique (Maddieson, 1991b). 
Table 5a. Qualités vocaliques de la table $5 \mathrm{~b}$

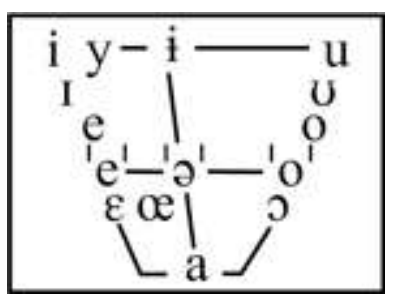

Table $5 \mathrm{~b}$. Occurrences des systèmes vocaliques par nombre de voyelles, de gauche à droite : du plus fréquent au moins répandu. La voyelle centrale qui relève probablement d'une autre dimension est représentée par un cercle transparent.

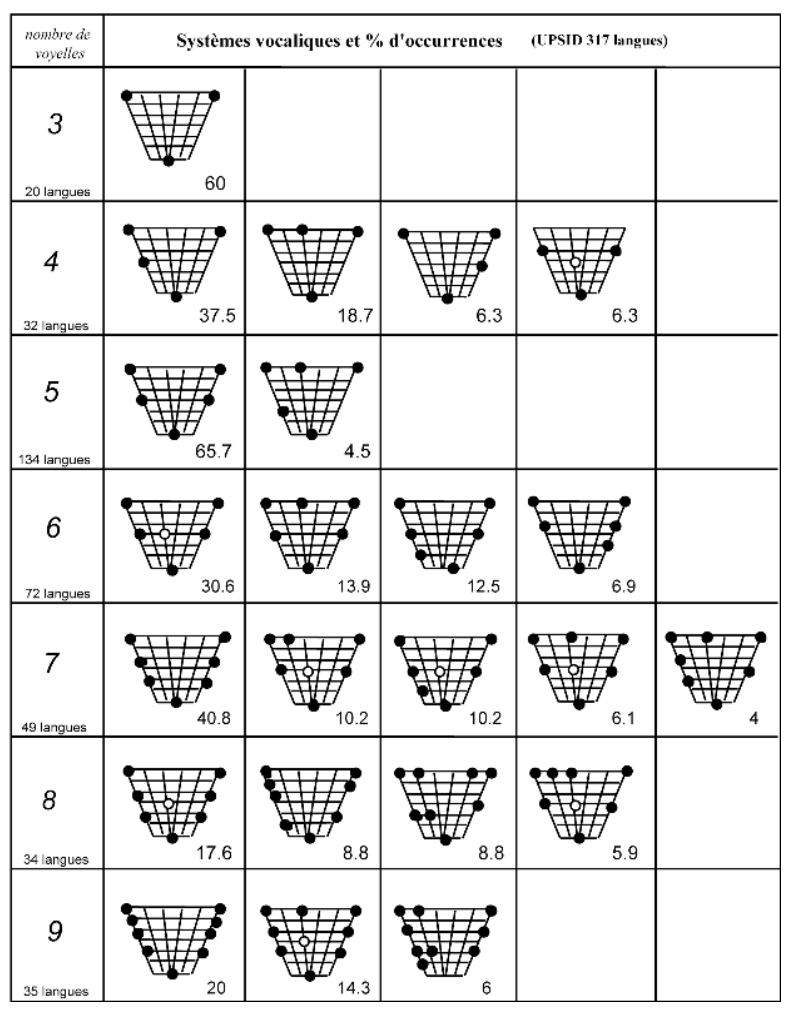

Parmi les systèmes pairs à 6 et 8 voyelles, les plus fréquents possèdent une voyelle centrale de type /ə/ qui pourrait renvoyer à une autre dimension liée probablement aux principes intrinsèques de la réduction vocalique (Schwartz \& al., 1997a). À 9, le système préféré est, comme le système à 7 , constitué de voyelles situées à la périphérie. Dans ces systèmes, la symétrie entre voyelles d'avant et voyelles d'arrière est une tendance forte. Dans les cas d'asymétrie (30\% des systèmes d'UPSID) les périphériques antérieures sont très souvent plus nombreuses que les postérieures (seulement $9 \%$ des langues ont plus de voyelles périphériques à l'arrière).

Au-delà de 9 timbres de base, il est clair que les langues puisent leurs unités distinctives dans d'autres dimensions. En effet, les systèmes de plus de 9 voyelles, vraisemblablement saturés, diminuent leur nombre de timbres de base en ajoutant un système "parallèle » (système secondaire vs. primaire) dans une autre dimension : la nasalité en général, ou la quantité. Cette relation tendancielle a été proposée par Vallée (1994) qui a quantifié la relation entre nombre de voyelles par langue et timbres de base. Les grandes tendances mises en évidence dans la structure des systèmes primaires se retrouvent dans les 
systèmes secondaires : préférences pour le système à 5 , la dispersion périphérique et symétrique, la voyelle centrale de type /ə/ qui, comme dans les systèmes primaires, ne semble pas « interagir » avec les autres voyelles du système.

\subsection{La Théorie de la Dispersion-Focalisation (TDF)}

Depuis Liljencrants \& Lindblom (1972) différentes hypothèses ont été avancées pour prédire et comprendre la structure sonore des systèmes vocaliques à partir de contraintes articulatoires et auditives. Les meilleurs résultats ont été obtenus avec la théorie de la dispersion adaptative (TD) de Lindblom (1986). Mais les prédictions réalisées présentent toujours un trop grand nombre de voyelles hautes non périphériques /i $* \mathrm{w} /$ entre /i/ et /u/, fait peu observé dans les langues naturelles (Vallée, 1994).

41 La théorie ICP de la dispersion-focalisation (TDF) (Schwartz \& al., 1989; Schwartz \& al., 1997b) permet de résoudre ce problème en mettant en compétition, avec le principe de distance entre voyelles, base de la TD, un principe gestaltiste de prégnance intravoyelle : la focalisation. Dans l'espace vocalique des formants, la proximité de deux résonances convergence articulatoirement produite autour d'un changement d'affiliation entre cavités-, a pour effet de renforcer l'énergie dans une zone du spectre, donnant ainsi à la voyelle une qualité « focale » que l'on peut comparer à celle des couleurs qualifiées ainsi (Brown \& Lenneberg, 1954), sur lesquelles s'accordent les perceptions des sujets de différentes langues (Rosch-Heider, 1972).

La TDF fait donc l'hypothèse que, pour un nombre de voyelles donné $n$, le système phonologique préféré (c'est-à-dire le plus fréquent dans une base représentative des langues du monde) est obtenu par minimisation d'un coût d'énergie global :

$$
\mathrm{E}_{\mathrm{DF}}=\mathrm{E}_{\mathrm{D}}+\alpha \mathrm{E}_{\mathrm{F}}
$$

avec la composante de dispersion structurelle :

$$
E_{D}=\Sigma \Sigma(1 / \text { dij }) 2 \quad(i=1 . . n-1 \quad j=i+1 . . n)
$$

et la composante locale de focalisation $\mathrm{E}_{\mathrm{F}}$ pondérée par le paramètre $\alpha$ :

$$
\begin{aligned}
& E_{F}=\left(E_{12}+E_{23}+E_{34}\right) \text { avec } \\
& E_{f f+1}=-\Sigma \Sigma 1 /\left(\mathrm{F}_{f+1}^{i}-F_{f}^{i}\right)^{2} i=1 . . n f=1 . .3
\end{aligned}
$$

Les voyelles $\mathrm{V}^{\mathrm{i}}$ sont décrites par 4 formants $\left(\mathrm{F}_{4}\right.$ étant fixé à $\left.3650 \mathrm{~Hz}\right)$ exprimés en barks selon la formule proposée par Schroeder \& al. (1979) : bark = 7 ArgSh (Hz / 650).

Pour calculer la distance perceptive $d_{i j}$ entre deux voyelles $V^{i}$ et $V^{j}$, le modèle utilise une distance euclidienne sur $\mathrm{F}_{1}$ et $\mathrm{F}_{2}{ }_{2}$. Le second formant perceptif est évalué à partir de $\mathrm{F}_{2}, \mathrm{~F}_{3}$ et $\mathrm{F}_{4}$ sur la base d'un modèle qui a été introduit par Fant et développé par la suite (Schwartz, 1987). Nous avons introduit un poids $\lambda$ de $\mathrm{F}_{2}{ }_{2}$ par rapport à $\mathrm{F}_{1}$ :

$$
\mathrm{d}_{\mathrm{ij}}=\left[\left(\mathrm{F}_{1}^{\mathrm{j}}-\mathrm{F}_{1}^{\mathrm{i}}\right)^{2}+\lambda^{2}\left(\mathrm{~F}_{2}{ }^{\mathrm{j}}-\mathrm{F}_{2}{ }^{\mathrm{i}}\right)^{2}\right]^{1 / 2}
$$

pour tenir compte de la dilatation du triangle vocalique dans le sens de $\mathrm{F}_{1}$ par rapport à $\mathrm{F}_{2}$ (Lindblom, 1986), que l'on pourrait attribuer soit aux mécanismes psycho-acoustiques de masquage des aigus par les graves, soit aux différences de capacités psychomotrices du contrôle vertical de la mandibule par rapport au déplacement avant-arrière de la langue (Lindblom \& Lubker, 1985).

La TDF se différencie donc de la TD par l'introduction d'un deuxième coût qui diminue l'énergie des systèmes pour lesquels les voyelles ont des formants $F_{1}$ et $F_{2}, F_{2}$ et $F_{3}$ ou $F_{3}$ et $\mathrm{F}_{4}$ rapprochés - voyelles « focales »-ce qui les rend plus stables. 


\subsection{Résultats : les espaces des phases des structures vocaliques}

46 La TDF est paramétrisée par $\lambda$ et $\alpha$ qui renvoient à des propriétés externes, au sens où nous les avons définies dans le dernier paragraphe de la première partie. Pour tester leurs valeurs nous avons adopté une méthode utilisée notamment en chimie : la détermination de l'espace des phases (Schwartz \& al., 1995). Les différentes zones des espaces de phase ont été explorées extensivement, en particulier, grâce à une étude utilisant une méthode d'optimisation par algorithme génétique (Fargetton, 1993). Il s'est agi de trouver une zone $\lambda$ et $\alpha$ permettant de prédire les systèmes préférentiels de 3 à 9 voyelles (cf. par exemple la Figure 4 pour les systèmes à 5). Les résultats montrent que cette zone existe, et qu'elle est délimitée par :

$0.2 \leq \lambda \leq 0.3$ et $0 \leq \alpha \leq 0.4$

avec, pour assurer la stabilité des systèmes comportant la voyelle /y/, une contrainte supplémentaire sur $\alpha: 0.3 \leq \alpha \leq 0.4$. 
Figure 4. L'espace des phases présente en fonction des paramètres $\lambda$ (poids de $F_{1}$ par rapport aux autres formants) et a (poids de la focalisation par rapport à la dispersion) les 4 zones dans lesquelles se répartissent au sens de la TDF les systèmes à 5 qualités vocaliques.

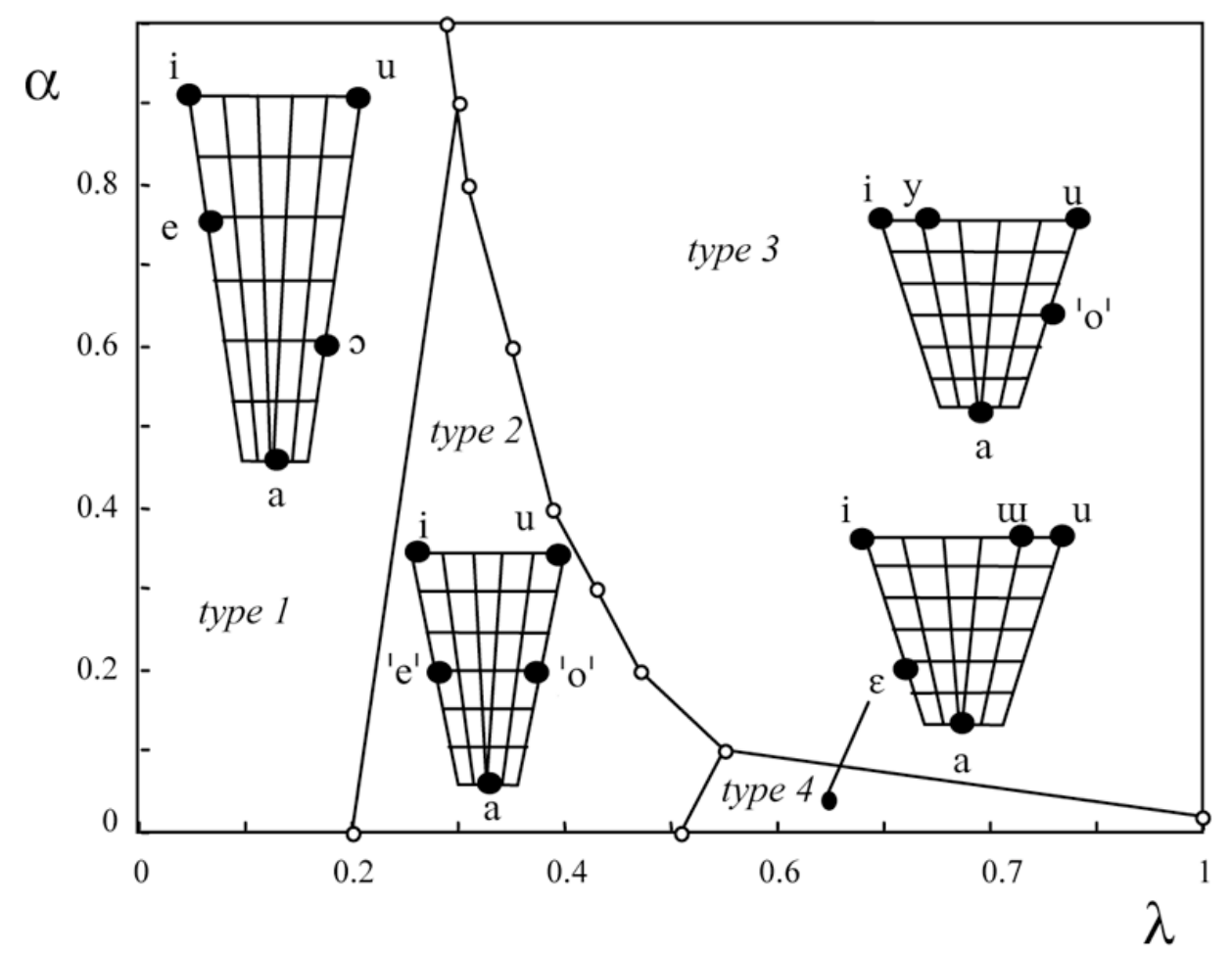

Dans la zone délimitée par $0.2 \leq \lambda \leq 0.3$ et $0 \leq a \leq 0.4$, le modèle TDF prédit effectivement le système à 5 , le plus fréquent des langues du monde.

C'est aussi la zone qui permet de prédire les systèmes préférés à 3, 4, et 7 qualités vocaliques.

- Liste des langues d'UPSID (451) par zone (le cardinal des systèmes est indiqué lorsqu'il est supérieur à 5):

/i e a $\supset \mathrm{u} /, 1$ langue : Kharia (10)

/i 'e' a 'o' u/, 92 langues:

Aïnou, Amharique, Arabela, Arménien de l'est, Ataya (17), Basque, Batak, Bedja, Bembe (10), Berta, Bourouchaski, Bribri (10), Burera, Caraïbe insulaire (10), Chatino (9), Chipewyan (14), Chuave,

Dagomba, Dakota (8), Daribi (10), Espagnol, Fasu (10), Garo, Géorgien (6), Grec, Hamer (10), Hawaïen, Hébreu, Japonais, Jingpo, Kadugli, Kaffa, Kaliai, Kewap (6), Khasi, Koiari, Kota, Kouroukh (10), Kullo, Kunjen, Luiseno, Luvale, Mabuiag, Maidu, Malayalam, Maori, Maung, Mixtèque (10), Moundari, Muinane, Mursi, Nambiquara du sud (18), Nasioi, Néo-araméen (16), Nere, Nez-percé, Ngizim (6), Noutka, Nubien, Pomo du sud-est, Quechua, Quiché, Roro, Rotokas, Russe, Séona (10), Songhaï (9), Suena, Swahili, Tai-shan, Tamang (10), Tarasque, Teke, Telefo (I8), Tiddim-Chin, Tlingit, Tucano (10), Tzeltal, Wappo, Wik-Munkan, Wintu, Wiyot, Yagaria, Yana, Yaqui, Yareba, Yourake (8), Yulu, Zhu /Oase (24), Zoque (6), Zoulou, Zuni.

/i y a o u/, 1 langue : Papago

li $\varepsilon$ a $u$ u/, 2 langues : Acoma, Cofan

On retrouve bien une valeur de $\lambda$ voisine de celle mise en évidence par les tests de DME ( Direct Magnitude Estimation) de Lindblom \& Lubker (1985). Si la focalisation semble une propriété du système auditif liée à des propriétés générales d'intégration (Schwartz \& Escudier, 1989), il reste à justifier - psycho-physiquement, informationnellement et/ou cognitivement - la valeur de la pondération $\alpha$, c'est-à-dire le poids de la focalisation par rapport à la dispersion. 


\section{Conclusion et perspectives} structures sonores. Les connaissances phonétiques permettent ainsi de fixer les contraintes naturelles que doivent respecter les théories phonologiques qui doivent satisfaire au souci de vraisemblance neurophysiologique.

\section{Dettes et reconnaissance}

Nos remerciements à Ian Maddieson qui a mis à notre disposition $\mathrm{UPSID}_{451}$ et nous a consacré beaucoup de son temps pour le suivi de son implantation. Un grand merci à Noureddine Abbadeni, Valérie Alcantara, Florence Mistrulli, Élisabeth Pinto pour leurs contributions. Une partie de cette présentation a été élaborée dans le cadre de la convention DGA/DRET $N^{\circ}$ 95/118 : Discrimination Multilingue Automatique, et financée par une action de la Région RhôneAlpes (ARASSH) et du GIS des Sciences de la Cognition. 


\section{BIBLIOGRAPHIE}

ABBADENI N. (1996) Tendances universelles et diversité des structures sonores des langues du monde. DEA Sciences du Langage, Université Stendhal, Grenoble.

ALCANTARA V. (1998) Le trait de labialité dans les systèmes vocaliques d'UPSID : de la typologie à la modélisation articulatoire. Application à la correction phonétique. TER de maîtrise Sciences du Langage, Université Stendhal, Grenoble.

BAUdoin de Courtenay J. (1894). An Attempt at a Theory of Phonetic Alter Nations. A Baudoin de Courtenay Anthology, Bloomington, Indiana, 144-212, édition de 1972 (article publié en polonais en 1894).

BROWN R.W., LENNEBERG E.H. (1954) A Study in Language and Cognition. J. Abnormal and Social Psychology 49, 454-462.

BоЁ L.J. (1997) Sciences phonétiques et relations forme/substance : 2 . Du poids de la substance sur la forme aux réarticulations scientifiques. Histoire Épistémologie Langage XIX, 2, 5-25.

BOYSSON-BARDIES DE B. (1996) Comment la parole vient aux enfants, Odile Jacob, Paris.

CREISSELS D. (1994) Aperçus sur les structures phonologiques des langues négro-africaines, 2 ème éd., ELLUG, Grenoble, 322 p.

CROTHERS J. (1978) Typology and Universals of Vowel Systems. Universals of Human Language, J.H. Greenberg Ed., 93-152, Stanford University Press, Stanford.

DAVIS B.L., MACNEILAGE P.F. (1994) Oganization of babbling : A case study, Language and Speech, 37 (4), 341-355.

FARGETTON L. (1993) Prédiction des systèmes vocaliques par algorithmes génétiques. Stage DUT, IUT Informatique Grenoble 2, ICP.

GREENBERG J.H., FERGUSON C.A., MORAVCSIK E.A. (Ed.) (1978) Universals of Human Languages : Method and Theory, Phonology, Word Structure, Syntax. Stanford Univ. Press, California.

HAGÈGE C. (1982) La structure des langues. Que sais-je, PUF, Paris. 2e édition 1986.

HOCKETT C.F. (1955) A Manual of Phonology. Waverly Press, Baltimore, 246 p., aussi Publications in Anthropology and Linguistics, Indiana University, Bloomington.

JAKOBSON R. (1941) Kindersprache, Aphasie, und allgemeine Lautgestze. Uppsala Universitets Årsskrift 1942, 1-83. Republié dans Jakobson R. (1962), Selected writings I, Mouton, The Hague, 328-401.

KEATING P.A. (1990) Coronal places of articulation. UCLA WPP 74, 35-60.

LAVER J. (1994) Principles of Phonetics. Cambridge University Press, Cambridge.

LADEFOGED P. (1995) The sounds of disappearing languages. The newsletter of The Acoustical Society of America, 5, 1, 1-6.

LADEFOGED P., MADDIESON I. (1996) The Sounds of World's Languages. Blackwell publishers, Oxford.

LINDBLOM B.E.F. (1986). Phonetic Universals in Vowel Systems. Experimental Phonology, Ed. by Ohala J.J., Academic Press, Orlando, Floridia, 13-44. 
LILJENCRANTS J., LINDBLOM B. (1972). Numerical Simulation of Vowel Quality Systems : the Role of Perceptual Contrast. Language 48, 839-862.

LINDBLOM B., LUBKER J. (1985) The Speech Homonculus : A Problem of Phonetic Linguistics. Phonetic Linguistics. V.A. Fromkin (Ed.), Academic Press, Orlando, Florida, 169-192.

LINDBLOM B., MADDIESON I. (1988) Phonetic universals in consonant systems. Language, Speech and Mind, ed. by Hyman L.H. \& Li C.N., London, New-york, 62-78.

LOCKE J.L. (1983) Phonological acquisition and change. Academic Press, New-York.

LOCKE J.L., PEARSON D.M. (1992) Vocal Learning and the Emergence of Phonological Capacity. A Neurobiological Approach. Phonological Development. Models, Research, Implications. C. Ferguson, L. Menn \& C. Stoel-Gammon. (Eds.) Timomium, Maryland, 91-129.

MACNEILAGE P.F., DAVIS B.L. (1990) Acquisition of speech production : Achievement of segmental independence. Speech Production and Speech Modeling, ed. by W.I. Hardcastle \& A. Marchal, Kluwer, Dordrecht.

MACNEILAGE P.F. (1998) The Frame/Content theory of evolution of speech production, Behavioral and Brain Sciences, 21,4, 499-511.

MADDIESON I. (1986) Patterns of Sounds. 2nd Ed. Cambridge University Press, Cambridge.

MADDIESON I. (1991a) Investigating Linguistic Universals. 12e Congrès International des Sciences Phonétiques, Aix-en-Provence, France, Vol. 1/5, 346-354, aussi UCLA Working Papers in Phonetics 78, 26-37.

MADDIESON I. (1991b) Testing the Universality of Phonological Generalizations with a Phonetically Specified Segment Database : Results and Limitations. Phonetica 48, 193-206.

MADDIESON I., PRECODA K. (1990) Updating UPSID. UCLA Working Papers in Phonetics 74, 104-111.

MAWASS K. (1997) Synthèse articulatoire des consonnes fricatives du français. Thèse de doctorat, INPG, Grenoble.

MILLER G.A. (1956) The magic number seven, plus or minus two : some limits on our capacity for processing information. Psychologycal Review 3, 81-97.

OHALA J. (1983) The origin of sound patterns in vocal tract constraints. The Production of Speech, ed. by P.F. MacNeilage, Springer Verlag, New-York, 189-216.

ROBB M., BLEILE K. (1994) Consonant inventories of young children from 8 to 25 months. Clinical Linguistics and Phonetics, 8, 295-320.

ROSCH-HEIDER E. (1972) Universals in Color Naming in Memory. J. Experimental Psychology 93, 10-20. RUHLEN M. (1987) A Guide to the World's Languages. Volume 1. Classification. Edward Arnold, London.

SCHROEDER M.R., ATAL B.S., HALL J.L. (1979) Objective Measure of certain Speech Signal Degradations based on Masking Properties of Human Auditory Perception. Frontiers of Speech Communication Research, B. Lindblom, S. Ohman, (Eds.) 217-229. London : Academic Press.

SCHWARTZ J.L. (1987) Représentations auditives des spectres vocaliques. Thèse de Docteur es Sciences Physiques, INP Grenoble.

SCHWARTZ J.L., BOË L.J., PERRIER P., GUÉRIN B, ESCUDIER P. (1989) Perceptual Contrast and Stability in Vowel Systems : A 3-D Simulation Study. Eurospeech 1, 63-66. 
SCHWARTZ J.L., BOË L.J., VALLÉE N. (1995) Testing the Dispersion-Focalization Theory : Phase Spaces for Vowel Systems. XIIIth Int. Congr. of Phonetic Sciences, 1, 412-415.

SCHWARTZ J.L., BOË L.J., VALLÉE N., ABRY C. (1997a) Major Trends in Vowel System Inventories. J. of Phonetics 25, 233-253.

SCHWARTZ J.L., BOË L.J., VALLÉE N., ABRY C. (1997b) The Dispersion-Focalization Theory of Vowel Systems. J. of Phonetics 25, 255-286.

SCHWARTZ J.L., ESCUDIER P. (1989) A Strong Evidence for the Existence of a Large Scale Integrated Spectral Representation in Vowel Perception. Speech Communication 8, 235-259.

SEDLAK, P. (1969). Typological Considerations of Vowel Quality Systems. Working Papers on Language Universals 1, Stanford University, 1-40.

STEFANUTO M. (1996) Typologie des lieux d'articulation des langues du monde. TER Sciences du Langage, Université Stendhal, Grenoble.

STEFANUTO M. (1997) Apport de la neurocognition comparée, développementale et pathologique dans l'étude des tendances universelles phonologiques. DEA Sciences du Langage, Université Stendhal, Grenoble.

Stoel-Gammon C. (1985) Phonetic Inventories 15-24 months : A longitudinal study, J. of Speech and Hearing Research, 23, 506-512.

TROubEtzKoy N.S. (1939). Grundzüge des Phonologie. Travaux du Cercle Linguistique de Prague 7, 272p., traduit en français par Cantineau J. (1970) sous le titre Principes de phonologie, Klincksieck, Paris, 394p.

VALLÉE N. (1994) Systèmes vocaliques : de la typologie aux prédictions. Thèse de Doctorat Sciences du Langage, Université Stendhal, Grenoble.

VALLÉE N., BOË L.J., STEFANUTO M. (1998) Les systèmes consonantiques. Des tendances universelles à

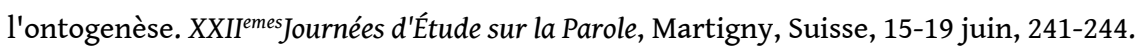

\section{AUTEURS}

\section{NATHALIE VALLÉE}

Institut de la Communication Parlée, Université Stendhal - Grenoble III - INPG

\section{LOUIS-JEAN BOË}

Institut de la Communication Parlée, Université Stendhal - Grenoble III - INPG

\section{MURIEL STEFANUTO}

Institut de la Communication Parlée, Université Stendhal - Grenoble III - INPG 